\title{
La mise à nu du moi malade chez Hervé Guibert
}

\section{The Revealing of the Inner Self in Hervé Guibert's Novels}

\author{
Anca Porumb \\ Université Babeş-Bolyai, Cluj-Napoca \\ ancaporumb76@yahoo.com
}

\begin{abstract}
In the middle of AIDS explosion during the 80 's, Hervé Guibert is one of the victims of this unexpected and unknown illness even for the medecine. He is not an ordinary sick person but he is the writer revealing himself completely and considering writing the only way for struggling against AIDS, against himself in order to reborn as a new spirit thanks to the power of words.
\end{abstract}

Key-words

AIDS, illness, writing, medecine, identity.

\section{Resumen}

Mientras la enfermedad hace su aparición en los años 1980, Hervé Guibert aparece como una de las víctimas de una afección muy extraña todavía en el mundo de la medicina. Pero no es simplemente una persona infectada, Guibert es el escritor sincero del sida, habiendo escogido las palabras como su único aliado por su proyecto de volver a nacer con un nuevo yo que no morirá nunca en la memoria de la gente.

\section{Palabras clave}

sida, enfermedad, escritura, medicina, identidad. 


\section{Introduction}

Le début des années 1990 en littérature est marqué par la parution des romans $\grave{A}$ l'ami qui ne m'a pas sauvé la vie et Le Protocole compassionnel de Hervé Guibert, romans controversés vu que l'écrivain ne se contente plus d'écrire à propos de la maladie du XXe siècle, qui est le sida. Il choisit de pousser l'autobiographie plus loin, sans penser peut-être au changement de l'horizon d'attente des lecteurs, qui se trouvent, aux carrefours des XXe et XXIeme siècles dans la position des témoins d'une littérature des confessions différente de celle dont parlait J.J. Rousseau ${ }^{1}$. Se replier sur soi-même et aller jusqu'au plus intime de son âme et de son corps, ce sont les directions principales des écrivains pour qui la maladie n'est plus un tabou.

Le thème que nous avons choisi de développer dans cette étude nous oblige à clarifier d'abord certains aspects à l'égard de ce qui s'appelle littérature du sida ou roman du sida. Dans quel contexte le roman est-il né? Pourquoi la maladie a-t-elle joué un rôle si important dans la littérature?

Nous passons ensuite en revue les théories à l'égard du genre littéraire guibertien, en insistant davantage sur l'autofiction comme terme qui définit le mieux les romans de Hervé Guibert, pour arriver finalement aux trois aspects caractéristiques de son œuvre qui décrit, à la première personne, le sida dans les moindres détails, parce que l'unique désir de l'auteur est de "coucher le moi sur le papier" (Jean-Pierre Boulé, 2001: 285).

La première partie, La frustration du moi malade, nous dirige vers le tableau d'un monde médical où l'impossibilité des médecins de maîtriser la maladie les plonge dans un état de confusion et de compétition côtoyant le cynisme.

La deuxième partie, Âme et corps en dégradation, se penche vers l'esthétique du laid et sur la solitude écrasante qui anéantit le protagoniste en quête d'une identité arrachée par le sida.

La dernière partie de l'étude, La réécriture du moi, continuera la question de la lutte contre le destin cruel et la fin inévitable, ayant cette fois-ci les mots comme seule force capable de sauver une âme troublée et marginale, condamnée à la mort.

\section{Le contexte. Les enjeux}

\subsection{Le contexte}

D'abord, ce fut l'année 1968 celle qui a changé la conscience collective en ce qui concerne la sexualité, surtout à l'intérieur de la communauté homosexuelle aux États-Unis et en Grande-Bretagne. Le désir de ne plus se cacher a poussé les personnes gay à vivre intensé-

1 Nous citons les mots de Jean-Jacques Rousseau dans Confessions, Livre IV: “C'est à lui [le lecteur] d'assembler ces éléments et de déterminer l'être qu'ils composent: le résultat doit être son ouvrage; et s'il se trompe alors, toute erreur sera de son fait." 
ment leurs aventures sexuelles selon le slogan que nous essayons de résumer en "Jamais avec le même partenaire". Dix ans plus tard, vers la fin des années 1970, l'humanité entière était sous le choc d'une nouvelle épidémie qui ravageait ces communautés, elles-mêmes jetées dans une confusion côtoyant la panique. Une épidémie dont le narrateur du roman À l'ami qui ne m'a pas sauvé la vie parle de la "fameuse maladie":

C'est Bill qui le premier me parla de la fameuse maladie, je dirais en 1981. Il revenait des États-Unis où il avait lu, dans une gazette professionnelle, les premiers comptes rendus cliniques de cette mort particulièrement engendrée. (Guibert, 1990: 21)

L'année 1981 évoquée dans le roman situe dans le temps une réalité dure, celle de la première étape, selon Jeffrey Weeks ${ }^{2}$, des réactions envers la maladie:

A. Entre les années 1981-1983, ce fut le contact direct avec la crise du sida et de la mort.

B. La période entre 1983-1986 se caractérise par la panique morale où les personnes contaminées se découvrent victimes de la marginalisation.

C. Après 1986, le sida devient une question autant politique que médicale et les campagnes de prévention s'intensifient.

C'est dans ce contexte que la littérature voit naître un nouveau genre, celui du roman du sida. Jean-Pierre Boulé, spécialiste de l'oeuvre de Hervé Guibert, nous offre "une archéologie des écrits du sida en France de 1985 à 1988" (p. 14), période qui coïncide avec l'attitude de rejet à tous les niveaux: médical, social, politique et même personnel, car les familles ayant un membre infecté par le virus préfèrent le silence et l'isolement.

Bien que Jean-Pierre Boulé affirme que les premiers livres sur le sida ont des auteurs plutôt inconnus ${ }^{4}$, nous rappelons ici le roman La Gloire du paria de Dominique Fernandez, paru en 1987, qui se détache de tous les autres récits et qui s'inscrit parfaitement dans la lignée des idées lancées par J. Weeks: "In AIDS such fears found a potent modern symbol." (J. Weeks, 2014: 382).

\subsection{Les enjeux}

Les formes que les romans du sida prennent (témoignage, journal, confession, autobiographie) ont, à notre avis, des raisons bien précises. Ainsi distinguons-nous deux grandes périodes dans l'évolution des récits de l'homosexualité et le sida.

2 Voir le chapitre 14 "Personal politics and moral conservatism" du livre de Jeffrey Weeks, Sex, Politics and Society, 3e édition, New York, Routledge, 2014, 357-390.

3 Jean-Pierre Boulé, "Typologies des premiers livres publiés en France sur le sida" in Littérature et sida, alors et encore, Brill /Rodopi, vol. 62, 2016, 14-26.

4 Il mentionne Hélène Laygues, Jean-Paul Aron, Alain Emmanuel Dreuilhe. 
Au début, il y avait le désir de sortir de l'anonymat et de la clandestinité en faisant de la maladie le symbole nécessaire pour rendre les personnes homosexuelles fières de leur choix. Tel est le récit de Dominique Fernandez évoqué plus haut, où les personnages Marc et Bernanrd regardent leur couple comme une œuvre d'art et le sida comme la perfection atteinte grâce à l'homosexualité.

Ensuite, les auteurs se sont mis au service de l'humanité en tirant un signal d'alarme sur la gravité de la maladie. Cela correspond à l'étape de la panique qui envahit les milieux gay et Hervé Guibert réussit à peindre le portrait d'un malade de sida dans les mondres détails, en s'appropriant à la fois une identité personnelle et une autre collective.

Une fois la panique apaisée par l'évolution de la médecine, les années 1990 se caractérisent en littérature du sida par un changement du récit, qui n'envisage plus la maladie comme une menace mais comme un choix. Les partenaires ont maintenant la liberté de se protéger et de protéger les autres ou de vivre pleinement leur sexualité sans se soucier de la contamination. Ainsi les romans se transforment-ils en récits érotiques acquérant une dimension pédagogique. Les lecteurs sont les témoins des scènes fortes où la pudeur n'a plus de place. Dans un espace urbain privilégié, les partenaires se cherchent, se retrouvent et se quittent sans regrets, toujours en quête des nouveautés et des sensations extrêmes.

Le risque de la contamination existe, c'est pourquoi au-delà des scénarios érotiques qui abondent dans les romans, il y a la préoccupation des personnages d'éviter ou pas la maladie. Dans un jeu continuel entre éros et thanatos, des pages entières dans les livres décrivent les réactions des partenaires à l'égard de l'usage du préservatif. Le débat permanent témoigne d'un certain égoïsme de la part du partenaire actif qui refuse toute méthode de protection tandis que le partenaire passif - et souvent c'est la même personne changeant les rôlespréfère la protection. Voilà deux images du roman Plus fort que moi (1998) de Guillaume Dustan, qui rendent le mieux les pratiques sexuelles d'aujourd'hui: “J'ai retiré la capote [...] et ça m'a fait rebander de penser que je pouvais baiser comme ça, il ne s'était aperçu de rien [...]" (Dustan, 1998: 70), bien que quelques pages plus haut le même protagoniste ait une toute autre attitude:

Au lit je lui ai mis une capote /.../ et trente secondes plus tard le compte était bon. Je me suis retourné et là j'ai vu la capote sur le bord du lit, sans rien dedans, et j'ai dit T'as retiré la capote?, et il a dit Oui, j'aime pas ça, et je suis devenu vert. (Dustan, 1998: 61).

Une conclusion partielle que nous pouvons tirer après avoir décrit le contexte et les enjeux des récits du sida se concrétise autour de l'idée d'un parcours difficile et ambigu. Les communautés homosexuelles prennent encore le risque en connaissance de cause au nom du plaisir charnel que les moyens de se protéger leur interdisent. 


\section{Les techniques narratives du moi malade chez Hervé Guibert}

Bien que les romans de Hervé Guibert soient considérés par la plupart des critiques une autofiction, terme introduit par Arnaud Genon que nous développerons plus tard, la question de genre auquel appartiennent À l'ami qui ne m'a pas sauvé la ville et Le Protocole compassionnel restera à jamais une provocation.

Parmi les premiers à s'intéresser à l'œuvre guibertienne, Jean-Pierre Boulé définit ses romans comme des romans faux et cela arrive à cause (ou grâce) de l'incipit du roman À l'ami: “J'ai eu le sida pendant trois mois. Plus exactement, j'ai cru pendant trois mois que j'étais condamné par cette maladie mortelle qu'on appelle le sida. (Guibert, 1990: 12) L'attitude de l'auteur envers la maladie, le dévoilement brusque et sincère dès la première page de ce qu'il considère un roman (un autre détail qui bouleverse les critiques) conduit J.P. Boulé à voir dans les récits de Guibert un jeu entre la fiction et la réalité, où le je narrateur ment afin de mener à bonne fin son projet littéraire. Car Hervé Guibert est avant tout un intellectuel ayant travaillé dans la rédaction du Monde. Son désir d'écrire quelque chose d'unique à la veille des années 1990 mène l'écrivain vers le style franc à l'égard de son homosexualité et sa maladie. Quoi de plus choquant pourrait-il arriver à l'intérieur du récit à la première personne? Hervé Guibert réussit à attirer les lecteurs dans son projet qui prend la forme d'un journal intime, mais qui ne respecte aucune chronologie, parce que nous nous trouvons tantôt dans le passé tantôt dans le présent.

La citation de J.P. Boulé au début de son livre: “Quelque part, j’avais écrit dans mon journal, avant de savoir que j'étais malade: 'Mort de sida. Indication superbe d'une biographie."' (Jean-Pierre Boulé, 2001:11) nous avertit qu'avant de nous confier entièrement à son récit, il faut pousser notre recherche plus loin pour trouver l'explication de sa démarche controversée où tout paraît si clair à la surface.

Stéphane Grisi(1996), moins cité dans les travaux sur Guibert, parle de l'autopathographie pour définir le genre littéraire où l'on a affaire avec l'autobiographie d'une personne malade, même avant les autres critiques considérés déjà des spécialistes de l'œuvre guibertienne (Boulé, Genon, Sarkonak ${ }^{5}$ ). Le je narrateur dans les deux romans suit son parcours de malade de sida déchiré entre les hauts et les bas qui le plongent dans l'angoisse.

La découverte de la maladie, son évolution rapide et le manque d'un traitement qui puisse donner de l'espoir au malade opère une mutation dans l'écriture des romans. Il n'y a plus le narrateur au premier plan du récit, mais la figure centrale autour de laquelle se tisse la trame, c'est le sida, personnage principal pour la première fois dans un roman. Voilà la particularité de Guibert, qui rend son genre littéraire unique et difficile à la fois, en assurant le succès de ses romans.

L'affirmation de Stéphane Grisi "Guibert tient avant tout la chronique de son sida"

5 Ralph Sarkonak emploie le terme sida-autofiction pour définir les romans de Hervé Guibert. 
(Grisi, 1996: 151), tout en reconnaissant le pacte autobiographique dans les deux livres, enlève le doute en ce qui concerne le jeu du je dans le récit dont les autres critiques parlaient, théorie à laquelle nous acquiesçons à notre tour.

\section{L'autofiction}

Avant de poursuivre le chemin de la quête des traits caractéristiques du moi dans les romans guibertiens, il s'impose de prêter plus d'attention à la notion d'autofiction que la critique a pourtant choisie pour définir l'œuvre de Hervé Guibert.

L'aventure a commencé avec les célèbres cases aveugles de Philippe Lejeune (1975) au moment où il établit une nette différence entre le roman, qui est un univers de l'imaginaire et l'autobiographie, où l'auteur est présent dans le texte sans dissimuler son nom, en devenant le lien entre la réalité qu'il décrit et le texte qui naît de cette réalité. Les cases aveugles de Lejeune provoquent, deux ans plus tard, en 1977, Serge Dubrovsky ${ }^{6}$ à donner naissance à un terme nouveau, l'autofiction, un mot-valise qui perdra vite son statut de néologisme.

Chercheurs, critiques et écrivains à la fois se sont lancés dans des polémiques à l'égard de l'identité du je du récit, nous l'avons déjà remarqué. Parmi les nombreuses théories et définitions qu'on a attribuées à l'autofiction, nous en avons choisi deux.

En respectant la chronologie, la première est celle de Vincent Colonna, qui, dans sa thèse de doctorat, considère l'autofiction comme "une œuvre littéraire par laquelle un écrivain s'invente une personnalité et une existence, tout en conservant son identité réelle." (Colonna, 1989: 30) La deuxième définition appartient à Marie Darrieussecq pour qui "l'autofiction est un récit à la première personne, se donnant pour fictif (souvent on trouvera la mention roman sur la couverture) mais où l'auteur apparaît homodiégétiquement sous son nom propre et où la vraisemblance est un enjeu maintenu par des effets de vie.» (Darrieussecq, 1996: 369 ) Pourquoi ce choix parmi tant d'autres définitions? Parce que les deux surprennent le mieux la démarche de Hervé Guibert en ce qui est le rapport entre le je qui écrit et le je dont on écrit.

La quête du moi qui caractérise les romans guibertiens s'inscrit parfaitement dans la lignée des tendances du XXe siècle par l'écriture toujours à la recherche du moi intime. N'oublions pas que la littérature après 1945 a subi un déplacement. D'abord, c'est la littérature après Auschwitz quand l'homme découvre la difficulté de dire la vérité sur soi-même par le biais d'une fiction pure ainsi que par le biais de l'autobiographie. Ainsi l'autofiction lui offre-t-elle le confort de résoudre le conflit intérieur entre la mémoire et l'oubli. Ensuite, c'est l'année 1968 qui ouvre la voie vers la libération des mœurs, vers une sexualité intensément vécue. Malheureusement, cette libérté sexuelle ne tarde pas à engendrer des drames une fois l'épidémie de sida éclatée dans les communautés homosexuelles. Le récit du moi malade

6 Serge Doubrovsky emploie pour la première fois en 1977 le terme d'autofiction à la quatrième de la couverture de son roman Fils. 
se cherche un autre canevas qui lui permette un dévoilement total, une écriture sincère et qui oblige dorénavant les lecteurs à une réception différente de la littérature du moi.

Cela signifie, pour conclure notre petite incursion dans les théories de l'autofiction, de penser en tant que lecteurs à remplacer le pacte autobiographique de Philippe Lejeune par un autre type de pacte que Awatif Beggar appelle le pacte autofictif.

\section{La frustration du moi malade}

Quand il s'agit du sida, on a affaire à une maladie totale qui éloigne les personnes chères et oblige le malade à une existence solitaire, isolée, et ce qui est pire, au rejet des autres, famille et amis à la fois. La société entière vit dans l'ignorance et dans la peur d'une contamination imminente, alors il faut écarter la personne infectée pour se mettre à l'abri. Au moment où cette attitude "contamine" les médecins, le drame augmente.

L'époque où Hervé Guibert se décide à dévoiler ses secrets, au début des années 1990, les hôpitaux en France sont assaillis par des personnes souffrantes d'une maladie inconnue. Les médecins se lancent vite dans une course dominée par l'ambition de trouver un vaccin qui leur apporte la gloire. Ainsi les cliniques se transforment-elles en endroits d'humiliation et d'aliénation en tant qu'individu.

Accusé d'exhibition, Guibert nous a laissé des témoignages importants à l'égard de la réalité médicale aux temps où le sida signifiait une double condamnation; d'un côté, le malade apprend à cohabiter avec une maladie qui mène à la mort; de l'autre côté, il fait face à l'arrogance des institutions médicales. ${ }^{7}$

D'abord, le roman À l'ami qui ne m'a pas sauvé la vie s'avère être plutôt un exercice ou une première de la maladie et de la mort. Guibert fait l'apprentissage du drame qui ne l'épargnera pas plus tard par le biais de son ami, Muzil, qui lui peint le tableau le plus sombre d'une réalité médicale en France des années 1980. La médecine tâtonnait toujours en soumettant les patients à des examens presque inhumains et inutiles. La méconnaissance du sida éveille des scénarios fous:

C'était l'époque où les bruits les plus fantaisistes, mais alors semblaient crédibles tellement on en savait peu sur la nature et le fonctionnement de ce qui n'avait pas encore été cerné comme virus, un lento ou un rétrovirus voisin de celui qui se tapit chez les chevaux, se propageaient sur le sida: qu'on l'attrapait en sniffant du nitrite d'amyle ${ }^{8}$, soudain retiré de la consommation, ou qu'il s'agissait de l'instrument d'une guerre biologique lancée tantôt par Brejnev tantôt par Raegan. (Guibert, 1990: 39).

7 Arnaud Genon le constate dans son livre Hervé Guibert. Vers une esthétique du postmoderne, Paris, l'Harmattan, 2007: "tout d'abord le virus du sida est en train de l'exterminer, de le ronger, tentant de le réduire à néant, mais à cela s'ajoute 'la moulinette administrative' qui, elle, nie l'identité du malade."

8 Le nitrite d'amyle est un composé chimique volatil et nocif, qui a des propriétés de vasodilatateur et a été d'abord utilisé comme médicament, ensuite comme drogue récréative dans les milieux festifs et sexuels. 
L'écrivain trouve la force, cinq ans plus tard, de remémorer les événements et les expériences vécus en tant que témoin et patient et de décrire les symptômes de la maladie comme s'il voulait laisser à la postérité infectée un guide médical qui puisse leur servir à comprendre l'évolution du sida. Les épisodes de fièvre violente, les ganglions, les abcès au fond de la gorge, la dégradation des reins, les prises de sang qui révèlent l'avancée rapide $\mathrm{du}$ virus et qui sont incontournables pour avoir accès au traitement avec $\mathrm{AZT}^{9}$ ne sont que quelques témoignages sincères d'une personne qui est désormais consciente de l'irréversible. Comme le début de la maladie est fulminant, Guibert a hâte de mettre sur le papier l'aller-retour continuel à l'hôpital. Le roman coïncide avec les premiers symptômes du sida et avec la découverte du personnage/narrateur de l'indifférence des médecins.

Au-delà de la compassion envers les autres, Guibert ne renonce jamais au ton révolté au moment où il est en présence des médecins:

Le médecin qui m'annonça mon résultat m'était antipathique, et j'accueillis bien sûr froidement la nouvelle, pour en finir le plus vite avec cet homme qui faisait son travail à la chaîne, trente secondes et un sourire et un prospectus pour les séronégatifs, de cinq à quinze minutes d'entretien 'personnalisé' pour les séropositifs. (Guibert, 1990: 147).

L'inconnu dans lequel cette indifférence plonge les patients conduit l'écrivain à gérer tout seul sa maladie. Il se lance dans l'analyse du sida en empruntant à la médecine le langage spécifique comme un vrai spécialiste fasciné et déçu en même temps:

Je suis très attentif aux manifestations de la progression du virus, il me semble connaître la cartographie de ses colonisations, de ses assauts et de ses replis, je crois savoir là où il couve et là où il attaque, sentir les zones encore intouchées [...] (Guibert, 1990: 45).

Le manque d'empathie dans les cliniques sera souligné dans les deux romans de Guibert et la frustration d'un moi condamné à subir les humiliations d'un système sourd aux souffrances des porteurs de ce nouveau virus augmente à chaque phase de la maladie. Après avoir connu les drames des malades qui avaient plutôt servi à des expériences scientifiques qu'au souci de la guérison, nous sommes en présence d'un Hervé Guibert menacé par l'écoulement du temps mais surtout par l'inexistence d'un traitement qui puisse prolonger sa vie.

Le roman Le Protocole compassionnel est le récit du désespoir d'un malade dans la phase finale de la maladie. Les premières pages du livre sont suffisantes pour se rendre compte du message: un médicament procuré sur le marché noir, le DDI ${ }^{10}$, reste le seul espoir devant l'indifférence des médecins. La déshumanisation atteint le comble dans une socié-

9 AZT: Zidovoudine, un antirétroviral actif contre le VIH, virus responsable du sida.

10 DDI: la didanosine est un antirétroviral, un inhibiteur nucléosidique de la transcriptase inverse (NRTI), utilisé dans le traitement du sida avec l'AZT. 
té plus malade que les personnes infectées et on apprend à quel point le désir de survivre change le comportement des gens:

Le garçon est mort le samedi, et c'est dans la nuit du dimanche au lundi que Jules est allé au Scorpio se faire livrer ces doses. Ainsi je tirais mes forces d'une substance qui était destinée à un mort. (Guibert, 1991: 19).

Ce qui est à remarquer c'est le sentiment de culpabilité qui s'empare du protagoniste et la frustration est encore plus forte qu'au début de la maladie.

Toujours fidèles à l'idée que l'écrivain ne cesse d'être déchiré entre un moi personnel et un autre moi collectif par la voie de tout dire pour laisser un témoignage, nous poursuivons notre analyse dans la direction d'un jeu cinématographique auquel Guibert nous provoque dans son récit.

\section{6. Âme et corps en dégradation}

Pour ce faire, le roman Le Protocole compassionnel continue à faire l'objet de notre intérêt parce qu'il y a dans ses pages un film qui se déroule devant les yeux des lecteurs, un film inquiétant qui suit le rythme soutenu de la maladie qui a hâte d'occuper et de maîtriser la vie de l'auteur. Miroir et caméscope deviennent une présence dans l'existence du protagoniste en état de confusion entre la proposition de son petit-ami, Jules, et les avertissements de Hector "On va dire que vous vous exhibez." (Guibert, 1991: 26)

Le morceau du texte qui s'étend sur les pages 25 et 26 du roman plonge le malade dans une nouvelle étape de sa maladie. Nous considérons la question "Photographier mon cadavre, ou alors photographier mon squelette vivant?” (Guibert, 1991: 26) le moment où Guibert passe d'un état d'inconscience à celui de la conscience de soi et de la réconciliation avec la maladie et la mort imminente:

J'avais retrouvé des textes, écrits quand j'avais vingt ans, qui décrivaient déjà ce spectacle, cette maladie et cette nudité. Mais là, saisi par la proposition de Jules, je n'en comprenais pas le sens: que voulait-il dire par squelette? (Ibid.)

Le mot "squelette" fait partie du champ lexical de la mort tandis qu'il est encore vivant. L'association des mots pourrait paraître bizarre, mais elle traduit le désir de l'auteur de bien délimiter un cadavre d'un squelette vivant qui n'a pas dit son dernier mot.

Il y a dans ce livre un Hervé Guibert très sage qui accepte tous les examens médicaux épuisants et douloureux. Ce qui l'intéresse maintenant c'est la maîtrise de son corps décrépit et la lutte avec son plus grand ennemi, le temps.

Le miroitement obsessif afin de suivre la dégradation continuelle de son corps est interprété par C. Herzlich et J. Pierret, dans Malades d'hier, malades d'aujourd'hui, comme 
une lecture du corps, qui est toujours "une construction: du corps lui-même et de ses rapports à la maladie." (Herzlich et Pierret, 1991: 101) On dirait qu'il s'agit chez Guibert d'un échec de cette lecture parce que plus il se regarde dans le miroir, plus il sent que le corps ne lui appartient plus:

Cette confrontation tous les matins avec ma nudité dans la glace était une expérience fondamentale, chaque jour renouvelée, je ne peux pas dire que sa perspective m'aidait à m'extraire de mon lit. Je ne peux pas dire non plus que j'avais de la pitié pour ce type, ça dépend des jours, parfois j'ai l'impression qu'il va s'en sortir puisque des gens sont bien revenus d'Auschwitz, d'autres fois il est clair qu'il est condamné, en route vers la tombe, inéluctablement. (Guibert, 1991: 15)

La souffrance physique transforme complétement la personne malade et Guibert en est bien conscient. C'est pourquoi il quitte bientôt la salle de bains qui était son espace préféré pour observer sa métamorphose. Rester cloué dans un seul endroit signifierait la fin rapide. La photo, qui est une image fixe, est remplacée par le film où il y a du dynamisme, car bouger est la certitude d'être encore vivant. Et la vidéo lui apporte le bonheur tant voulu et l'oubli de la maladie, ce qui est à remarquer dans son récit toujours alerte où toute une diversité de sentiments se mêlent: “j'ai l'impression d'être en lévitation et que mon crâne respire, je m'endors, je bande, je m'oublie, j'ai le fou rire, je perds toute notion du temps.' (Guibert, 1991: 100)

Après une longue pause dans la narration, on retrouve le personnage de nouveau à Paris, en train de se filmer pendant un autre examen, l'ultime cadre avant la fin du récit. Le roman finit par le même tourment de l'auteur qui ne cesse d'espérer jusqu'au dernier moment à un miracle de la médecine qui, hélas, reste encore à l'étape des expériences et des tests qui n'offrent aucune certitude aux séropositifs. Alors, Guibert continue son projet unique de surprendre en images vives son corps pour la postérité "Parce qu'il me semble suffisamment rare pour mériter qu'on en laisse une trace.” (Guibert, 1991: 225).

\section{La réécriture du moi}

Quand âme et corps sont pervertis par le virus meutrier, Guibert ressent le drame de la perte du contact avec sa propre personne, la perte de l'identité en tant qu'individu. Heureusement, le trait caractéristique des écrivains du sida est l'acharnement de tenir bon face à la maladie et de faire de l'écriture du moi malade un but dans le combat avec l'impuissance physique:

Écrire, c'est poursuivre une route qui mène à une destination donnée, c'est ne pas rendre larmes, armes et silence vaincu à la mort, c'est enfin rendre compte d'un hommage de plus à la vie en lui inventant un bout supplémentaire d'avenir, c'est tenir, s'en- 
têter à tenir le fil fragile des mots et des phrases, une manière de demeurer alors avec les autres. (Maxence, 1995: 25).

Nous avons choisi cette citation pour souligner encore une fois la démarche de l'écrivain de s'accrocher au monde et à la vie grâce aux mots. Quand la maladie est bel et bien présente dans l'existence du protagoniste, quand le virus s'installe de plein droit dans son corps comme le maître suprême, Hervé Guibert commence une lutte acharnée contre le sentiment de frustration qui le domine. Il ne s'agit pas de la frustration provoquée par son impuissance physique à laquelle il s'était déjà habitué. Nous saisissons un sentiment de rage venant de la frustration de ne plus être capable de maîtriser son écriture car son unique souci est dorénavant celui de raconter son histoire jusqu'à la fin de son livre et de sa vie:

Le livre lutte avec la fatigue qui se crée de la lutte du corps contre les assauts du virus. Je n'ai que quatre heures de validité par jour, une fois que j'ai remonté les stores immenses de la verrière, qui sont le potentiomètre de mon souffle déclinant, pour retrouver la lumière du jour et me remettre au travail. (Guibert, 1991: 66).

Hervé le narrateur ne cherche plus le miroir, les transformations subies par le corps ne l'intéressent pas parce qu'il se trouve dans une autre étape de sa vie où la fièvre de l'écriture dépasse la fièvre comprise au sens physiologique. L'auteur s'éloigne de l'aspect médical qui l'avait préoccupé auparavant pour se diriger vers une sphère supérieure, là où la maladie et la mort sont vaincues par les mots. Comme tout autre écrivain penché vers le récit du sida, Guibert trouve dans la maladie la source de survivre. La réalité cruelle qui ne cesse de le guetter, la mort qu'il côtoie à chaque instant sont vite munies de qualités positives; n'eût-été le sida, Hervé Guibert serait resté un anonyme parmi d'autres malades disparus.

La fureur de renaître avec un nouveau moi exorcisé des hantises de la fin certaine dévoile une personne entièrement abandonnée au pouvoir de l'écriture. Guibert n'a plus peur de la mort, ce qui lui fait peur est l'impossibilité de mener à bonne fin la guerre déclarée à la maladie. Le corps est vaincu par le sida mais peu importe, parce que c'est l'âme qui aura le dernier mot. L'auteur met toute la confiance dans le moi qui aura surgi après sa disparition physique, un moi sincère légué à la postérité avec l'espoir que celle-ci regardera dans les tréfonds de son âme pour s'apercevoir le tourment:

C'est mon âme que je dissèque à chaque nouveau jour de labeur qui m'est offert par le DDI du danseur mort. Sur elle je fais toute sorte d'examens, des clichés en coupe, des investigations par résonance magnétique, des endoscopies, des radiographies et des scanners dont je vous livre les clichés, afin que vous les déchiffriez sur la plaque lumineuse de votre sensibilité. (Guibert, 1991: 80).

Voilà le message et la quintessence des deux romans analysés. Au-delà de la révolte contre la famille, les amis, la médecine et la société, l'auteur engage un dialogue avec les 
lecteurs. Son ultime destination, plus privilégiée que les autres, se veut un appel à ceux pour qui il s'est donné la peine de tenir bon jusqu'à la naissance du livre. Cela est la certitude de son avenir dans la mémoire collective au moment où il arrive à ressentir le début de la dépersonnalisation du moi qui écrit car "C'est le DDI du danseur mort, avec le Prozac, qui écrit mon livre, à ma place." (Guibert, 1991: 84) Le conflit intérieur entre le moi malade et le moi qui naît en même temps que le texte rend le drame de la perte de son identité mais il rend aussi l'ambition de Guibert de reconquérir sa propre personne, son propre corps, son âme:

Je n'ai pas l'impression de ne plus être moi-même, ni d'être sorti de moi-même, ni d'être devenu un autre. Je suis le même qui pense pareil et qui l'écrit, auquel le médicament jusqu'à nouvel ordre donne l'énergie physique et morale de le faire (Ibid.: 84).

\section{Conclusion}

À travers cette étude, nous nous sommes posé la question de l'influence du sida sur le moi de Hervé Guibert dans ses deux romans, À l'ami qui ne m'a pas sauvé la vie et Le Protocole compassionnel.

Le tableau brièvement esquissé des échos de l'œuvre guibertienne nous a montré la complexité et l'originalité de sa narration, ce qui a donné beaucoup de fil à retordre aux critiques dans leurs efforts de définir son style unique de dire les choses. La diversité des études des romans de Hervé Guibert nous a convaincus de l'impact majeur de la maladie sur le récit du je. Le désir de tout dire à propos de sa propre personne entraîne toujours les lecteurs dans un jeu continuel entre fiction et réalité.

Nous avons suivi le parcours du je narrateur en tant que malade quand les rapports avec les médecins sont plutôt nuisibles, en tant qu'individu dans une société qui fait semblant de ne pas remarquer la présence d'un homosexuel ou en tant qu'ami trahi au moment même où il aurait pensé à l'appui des proches. Toutes ces hypothèses ont mené Guibert vers la quête d'une identité nouvelle, qui soit envisagée comme une naissance d'un autre moi réconcilié avec le monde et la maladie.

Au-delà du corps en décrépitude qui le rend physiquement impuissant, l'auteur a fait la découverte du pouvoir des mots. C'est à partir de ce moment-là que le protagoniste conscient de son destin commence la lutte au niveau du récit. Les blessures physiques et morales sont effacées pour laisser la place à l'écriture, car c'est l'écriture qui compte le plus dans son œuvre. Le caractère personnel et inouï de ses romans où tout un milieu interdit à l'époque se révèle à nos yeux et bouleverse l'ordre des choses. L'écriture du sida est peut-être née avec Hervé Guibert bien qu'il y ait eu avant des récits sur la maladie. Mais ce qui rend son écriture unique dans la nouvelle vague appélée roman du sida, c'est la volonté de l'auteur de choquer par la violence des mots et des images (il faut pas oublier sa passion pour la photo!). 
Ainsi tirons-nous la conclusion que le choix de se mettre à nu au nom de la vérité lui assure à jamais une place d'honneur dans la pléiade des écrivains sidéens.

\section{Références bibliographiques}

BEGGAR, Awatif. 2014. "L'autofiction: un nouveau mode d'expression autobiographique" in <www.revue-analyses.org>, vol. 9, N. 2, printemps-été 2014 [consulté le 12/05/19].

Blanckeman, Bruno. 2016. "Hervé Guibert, témoin d'exception" in Littérature et sida, alors et encore, Brill/Rodopi, vol. 62, 27-38.

Boulé, Jean-Pierre. 2001. Hervé Guibert: L’Entreprise de l'écriture du moi. Paris, L'Harmattan.

Boulé, Jean-Pierre. 2016. "Typologies des premiers livres publiés en France sur le sida" in Littérature et sida, alors et encore. Brill /Rodopi, vol. 62, 14-26.

Colonna,Vincent. 1989. L'Autofiction (essai sur la fonctionnalisation de soi en littérature), thèse de doctorat sur la direction de Gérard Genette [consulté le 16/02/19] <http://tel.archives-ouvertes.fr/docs/00/04/70/04/PDF/tel-00006609.pdf $>$.

DArrieussecQ, Marie. 1996. "L'autofiction, un genre pas sérieux" in Poétique, No. 107, septembre, 369-380.

Genon, Arnaud. 2007. Hervé Guibert. Vers une esthétique postmoderne. Paris, L'Harmattan.

Grisi, Stéphane. 1996. Dans l'intimité des maladies - de Montagne à Hervé Guibert, Paris, Desclée de Brouwer.

Guibert, Hervé. 1990. Á l'ami qui ne m’a pas sauvé la vie. Paris, Gallimard.

GuIBERT, Hervé. 1991. Le protocole compassionnel, Paris, Gallimard.

Herzlich, Claudine, Pierret, Janine. 1991. Malades d'hier, malades d'aujourd'hui, Paris, Payot.

Lejeune, Philippe. 1975. Le Pacte autobiographique. Paris, Editions du Seuil.

LÉvy, Joseph et Nouss, Alexis. 1994. Sida-fiction: Essai d'Anthropologie Romanesque, Lyon, Presses universitaires de Lyon.

LÉvy, Joseph, Quevillon, Lucie. 2010. "Sexualité et prévention dans les romans contemporains sur le VIH/sida" in Civilisations, 59-1.

Maxence, JeanLuc. 1995. Les écrivains sacrifiés des années sida. Paris, Bayard Editions.

Porumb, Anca. 2010. Homosexualité et sida. Essais sur le roman de Dominique Fernandez, Hervé Guibert et Yves Navarre. Cluj-Napoca, Casa Cărţii de ştiinţă.

Sarkonak, Ralph. 1997. Le corps textuel de Hervé Guibert. Paris, Lettres modernes, Minard. 
